\title{
The triple procedure: in the bag placement versus ciliary sulcus placement of the intraocular lens
}

\author{
Vincent M Borderie, Olivier Touzeau, Tristan Bourcier, Santos Carvajal-Gonzalez, \\ Laurent Laroche
}

\begin{abstract}
Aims-To evaluate the influence of intraocular lens (IOL) placement on triple procedure clinical results and to investigate whether it is appropriate to use phacoemulsification in patients with large lens nucleus.
\end{abstract}

Methods-40 consecutive penetrating keratoplasties combined with cataract extraction performed in a single institution were studied. Whenever possible a capsulorhexis was performed and the IOL was placed into the capsular bag. Phacoemulsification was used when the nucleus was too large to pass through the capsulorhexis.

Results-Out of 25 patients with an intact capsulorhexis phacoemulsification was used in $13(52.0 \%)$ whereas the entire nucleus passed through the capsulorhexis in the remaining 12 patients $(48 \%)$. The average 12 month visual acuity was 0.46 (SD 0.21) in patients with in the bag IOL $(n=23)$ and $0.29(0.08)$ in patients with ciliary sulcus IOL $(n=13)(p=0.04)$. Elevated intraocular pressure occurred in $26.1 \%(6 / 23)$ of patients with in the bag IOL and $61.5 \%(8 / 13)$ of patients with ciliary sulcus IOL $(p=0.08)$. The average postoperative graft thickness at 18 months was 552 (27) $\mu \mathrm{m}$ in the former group and $650(29) \mu \mathrm{m}$ in the latter group $(p=0.04)$. No significant difference in graft survival, postoperative endothelial cell density, astigmatism, and videokeratoscopic measurements was found between both groups.

Conclusion-In the bag placement of the intraocular lens during the triple procedure results in better outcome of transplantation than ciliary sulcus placement of the IOL. Phacoemulsification allows removal of large nuclei through a $5 \mathrm{~mm}$ capsulorhexis without performing relaxing incisions out towards the periphery of the capsule.

(Br F Ophthalmol 1999;83:458-462)

Extracapsular extraction is currently the gold standard of triple procedures combining penetrating keratoplasty, cataract extraction, and intraocular lens (IOL) placement. There is general agreement to place a posterior chamber IOL within the capsular bag rather than in the ciliary sulcus. ${ }^{12}$ To achieve this goal the capsulotomy can be performed with scissors or in a capsulorhexis fashion. With the former technique there is a tendency for the capsule to tear out towards the periphery if sharp junctions are present in the capsule, resulting in uncertain placing of the IOL. ${ }^{34}$ With the latter technique, the IOL can be placed securely within the capsular bag. However, performing a capsulorhexis is more difficult open sky than under a closed system as posterior vitreous pressure induces a tendency for the continuous tear capsulotomy to autoextend peripherally. This can often be avoided if a small size capsulotomy is performed but expression of large nuclei through a small capsular opening may be very difficult. ${ }^{3}$ We considered the use of phacoemulsification in patients with an intact capsulorhexis and large nucleus to achieve in the bag placement of the IOL.

The aim of the present study was to investigate whether it is appropriate to use phacoemulsification during a triple procedure in patients with large lens nucleus and to compare the results of the triple procedure and in the bag placement of the IOL with those of the triple procedure with ciliary sulcus placement of the IOL.

\section{Patients and methods}

We prospectively studied 40 consecutive penetrating keratoplasties combined with cataract extraction carried out in 40 patients between January 1993 and December 1996. These 40 triple procedures represented all of the triple procedures and $11.8 \%$ of the 340 penetrating keratoplasties performed at our institution during the 4 year study period. Whenever possible a continuous tear capsulotomy was performed and the intraocular lens was placed in the capsular bag. Phacoemulsification was used when the nucleus was too large to pass through the continuous tear capsulotomy without performing relaxing incisions out towards the periphery of the capsule.

Donor corneas were organ cultured as previously described. ${ }^{5}{ }^{6}$ Donor tissue characteristics are shown in Table 1 . All transplants were performed at a single institution by five surgeons. Two surgeons (LL, VMB) carried out $90 \%$ of the transplants. Out of 40 patients 19 (57.5\%) were operated on under general anaesthesia and $21(42.5 \%)$ under peribulbar anaesthesia. For the latter patients preoperative softening of the eye was performed using a balloon to minimise the posterior pressure. All donor buttons were punched from the posterior corneal surface by using the Hanna device. The average donor trephination size was 8.13 (SD $0.30) \mathrm{mm}$ (range $7.25-8.50 \mathrm{~mm}$ ). The Hanna corneal trephine system was used for trephination of recipient cornea. The average recipient 
Table 1 Patient and donor tissue characteristics

\begin{tabular}{|c|c|c|c|}
\hline & Overall $(n=40)$ & $\begin{array}{l}\text { Bag group } \\
(n=23)\end{array}$ & Sulcus group $(n=13)$ \\
\hline \multicolumn{4}{|l|}{ Preoperative diagnosis: } \\
\hline Fuchs' dystrophy & $11(27.5 \%)$ & $9(39 \%)$ & $2(15 \%)$ \\
\hline Keratoconus & $3(7.5 \%)$ & $3(13 \%)$ & $0(0 \%)$ \\
\hline Glaucomatous bullous keratopathy & $4(10.0 \%)$ & $2(9 \%)$ & $0(0 \%)$ \\
\hline Other corneal dystrophies & $5(12.5 \%)$ & $2(9 \%)$ & $4(31 \%)$ \\
\hline \multicolumn{4}{|l|}{ Corneal scar, corneal ulcer, } \\
\hline Trauma & $2(5.0 \%)$ & $1(4 \%)$ & $0(0 \%)$ \\
\hline Allograft rejection & $3(7.5 \%)$ & $1(4 \%)$ & $\begin{array}{l}1(8 \%) \\
\mathrm{p}=0.17(\mathrm{NS})^{\star \star}\end{array}$ \\
\hline \multicolumn{4}{|l|}{ Recipient rejection status: } \\
\hline Low risk & $24(60.0 \%)$ & $17(74 \%)$ & $6(46 \%)$ \\
\hline High risk ${ }^{\star}$ & $16(40.0 \%)$ & $6(26 \%)$ & $\begin{array}{l}7(54 \%) \\
\mathrm{p}=0.19(\mathrm{NS})^{\star \star} \\
\mathrm{p}=0.15(\mathrm{NS})^{\star \star \star \star}\end{array}$ \\
\hline Recipient age: & $9(22.5 \%)$ & $4(17 \%)$ & $3(23 \%)$ \\
\hline $28-60$ years & $14(35.0 \%)$ & $10(44 \%)$ & $2(15 \%)$ \\
\hline $61-70$ years & $17(42.5 \%)$ & $9(39 \%)$ & $8(62 \%)$ \\
\hline $71-82$ years & & & $\mathrm{p}=0.22(\mathrm{NS})^{\star \star}$ \\
\hline \multicolumn{4}{|l|}{ Donor tissue (mean (SD)): } \\
\hline Donor age (years) & $68(11)$ & $68(120$ & $\begin{array}{l}69(8) \\
\mathrm{p}=0.51(\mathrm{NS})^{\star \star \star}\end{array}$ \\
\hline $\begin{array}{l}\text { Endothelial cell density after } \\
\text { preservation }\left(\text { cells } / \mathrm{mm}^{2}\right)\end{array}$ & $2259(451)$ & $2330(498)$ & $\begin{array}{l}21176(401) \\
\mathrm{p}=0.40(\mathrm{NS})^{\star \star \star}\end{array}$ \\
\hline Preservation time (days) & $23.6(4.2)$ & $23.7(4.4)$ & $\begin{array}{l}23.5(4.2) \\
\mathrm{p}=0.84(\mathrm{NS})^{\star \star \star}\end{array}$ \\
\hline
\end{tabular}

${ }^{\star}$ High risk recipients were defined as having a vascularised cornea (two or more quadrants of corneal vascularisation) or a history of irreversible corneal allograft rejection.

$\star \star \chi^{2}$ test against bag group.

$\star \star \star$ Wilcoxon rank sum test against bag group.

$\star \star \star \star$ Fisher's exact test.

Table 2 Overall results ( $n=40)$. Visual acuity, astigmatism, videokeratoscopic measurements, intraocular pressure, graft thickness, and endothelial cell density were measured excluding patients with graft failure

\begin{tabular}{ll}
\hline Two year graft survival estimate (\%) & 66.3 \\
Spectacle corrected visual acuity at 12 months (mean (SD)) & $0.41(0.20)$ \\
Spectacle corrected visual acuity at 18 months (mean (SD)) & $0.43(0.20)$ \\
Subjective astigmatism (D) at 12 months (mean (SD)) & $4.04(1.93)$ \\
Subjective astigmatism (D) at 18 months (mean (SD)) & $3.53(1.44)$ \\
Videokeratoscopic astigmatism (D) (mean (SD)) & $4.87(3.29)$ \\
Predicted corneal acuity (mean (SD)) & $0.98(0.66)$ \\
Refractive power symmetry index (D) (mean (SD)) & $2.32(1.21)$ \\
Effective refractive power (D) (mean (SD)) & $45.75(2.51)$ \\
Maximum IOP (mm Hg) (mean (SD)) & $21.2(9.4)$ \\
Graft thickness at 12 months ( $\mu$ m) (mean (SD)) & $552(48)$ \\
Graft thickness at 18 months $(\mu \mathrm{m})$ (mean (SD)) & $576(52)$ \\
Keratoplasty to specular microscopy time (months) (mean (SD)) & $14(2)$ \\
Endothelial cell density (cells/mm ${ }^{2}$ (mean (SD)) & $1441(438)$ \\
Percentage of endothelial cell loss (mean (SD)) & $34.3(22.7)$
\end{tabular}

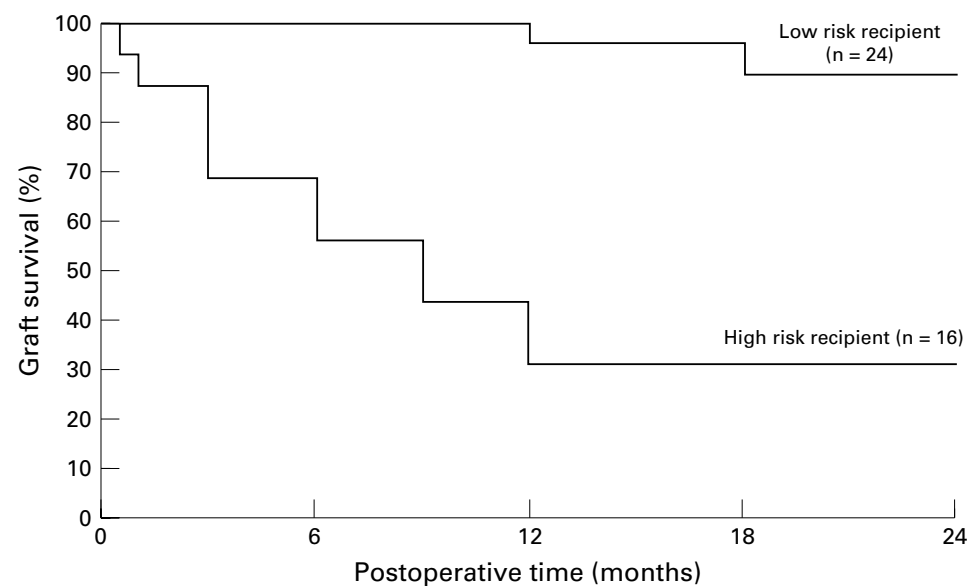

Figure 1 Influence of recipient rejection status on graft survival (Kaplan-Meier method). Log rank: $p<0.0001$. Multivariate Cox hazards model: $p=0.04$. The numbers of patients at risk at the beginning of the curves are indicated at the end of the curves.

trephination size was $7.88(0.29) \mathrm{mm}$ (range $7.00-8.25 \mathrm{~mm})$. A continuous tear capsulotomy was performed open sky using a cystotome and forceps. ${ }^{3}$ The target opening size was $5 \mathrm{~mm}$ to avoid tearing out towards the periphery. Hydrodissection was performed by injecting balanced salt solution under the anterior capsular edge which permitted small nuclei expression. If the nucleus was too large to pass through the capsulorhexis, phacoemulsification was used. The nucleus was fragmented into two or four pieces which were removed. Cortex removal was performed using the automated irrigation aspiration machine. The capsule was filled with sodium hyaluronate and a $5.5 \mathrm{~mm}$ or $6 \mathrm{~mm}$ PMMA posterior chamber IOL was introduced into the capsular bag. When posterior vitreous pressure was high and the capsule torn towards the periphery, the capsulotomy was done in a can opener fashion. The nucleus was then expressed and cortex was removed with the irrigation aspiration hand piece. A $7 \mathrm{~mm}$ PMMA posterior chamber IOL was inserted in the ciliary sulcus. If a tear in the posterior capsule occurred during the procedure, automated vitrectomy was performed and a $5.5 \mathrm{~mm}$ or $6 \mathrm{~mm}$ PMMA anterior chamber IOL or no lens was placed in the anterior chamber. Suture methods included 24 interrupted sutures, running suture, and a combination of interrupted and running sutures. Postoperatively, all patients were treated with topical dexamethasone and neomycin as previously described. ${ }^{6}$

The patient mean age was 65.8 (SD 13.0) years (range 28-82). Patient characteristics are shown in Table 1. High risk recipients were defined as having a vascularised cornea (two or more quadrants of corneal vascularisation) or a history of irreversible corneal allograft rejection.

Patients were hospitalised up to graft reepithelialisation. They were then examined at 2 weeks, 1, 3, 6, 9, 12, 18, 24, 30, 36, and 48 months after surgery. The average follow up time was 20.4 (9.5) months (range 12-48). At 12 months postoperatively all of the eyes were available for follow up. At 18 months 31 eyes $(77.5 \%)$ were available for follow up. The criteria for graft failure were irreversible graft stromal oedema or corneal opacification. We defined rejection as graft failure with rejection line, graft infiltrates, keratic precipitates, graft vascularisation, ciliary injection, or aqueous cells. The diagnosis of rejection was made only if the transplant had remained clear for an interval of at least 2 weeks after surgery.

During the second year following transplantation contact wide field specular microscopy (Konan Keeler Pocklington), ultrasonic pachymetry (DGH 1000) and corneal topography (EyeSys System 2000) were performed in clear transplants.

Categorical variables were analysed by the $\chi^{2}$ test or the Fisher's exact test. We studied unadjusted graft survival with the Kaplan-Meier method and compared the data with the log rank test. We then performed a multivariate Cox proportional hazards analysis, including variables that were significant at a univariate level $(\mathrm{p}<0.10)$. We studied the following variables: donor age, endothelial cell density after preservation, preservation time, recipient age, preoperative diagnosis, recipient rejection 


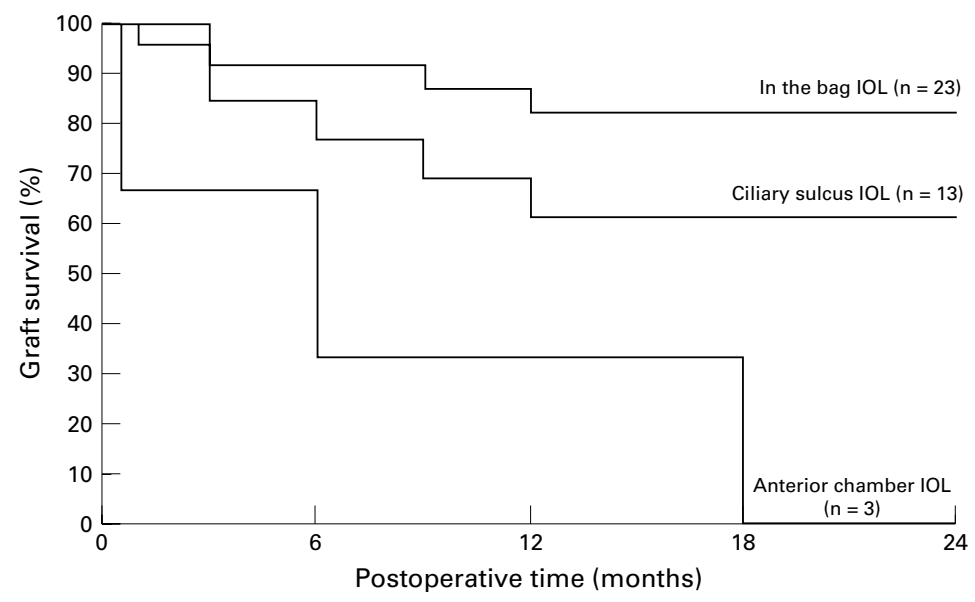

Figure 2 Influence of postoperative lens status on graft survival (Kaplan-Meier method). Log rank: $p=0.004$. Multivariate Cox hazards model: $p=0.12$. The numbers of patients at risk at the beginning of the curves are indicated at the end of the curves.

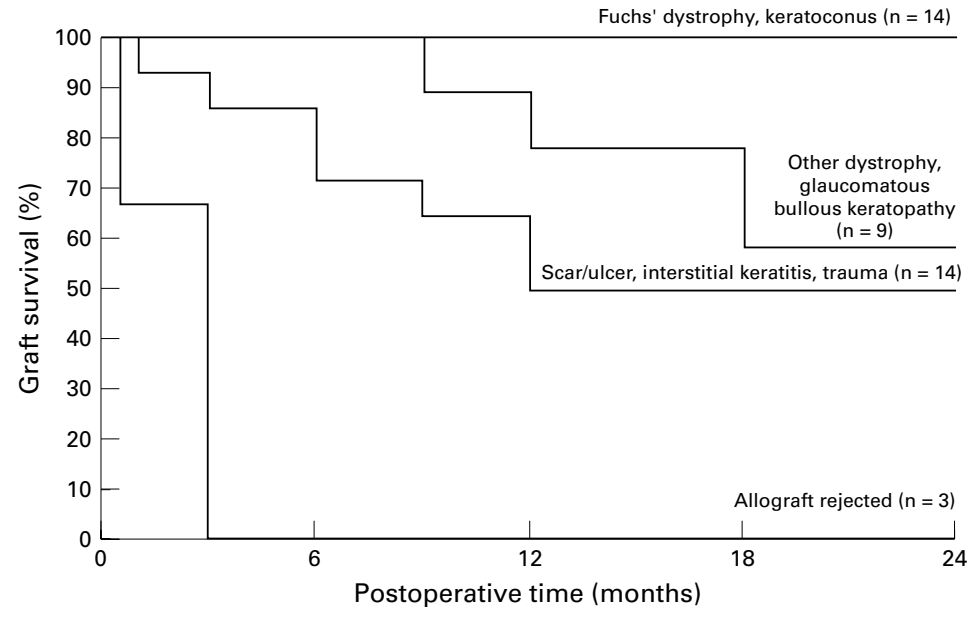

Figure 3 Influence of preoperative diagnosis on graft survival (Kaplan-Meier method). Log rank: $p<0.0001$. Multivariate Cox hazards model: $p=0.10$. The numbers of patients at risk at the beginning of the curves are indicated at the end of the curves.

status (high risk or low risk), preoperative intraocular pressure, donor trephination size, recipient trephination size, postoperative lens status, and suture method. Considering the high correlation among donor and recipient trephination size, we only included donor trephination size in the multivariate model. Donor tissue characteristics (that is, donor age, graft endothelial cell density after preservation, preservation time), visual acuity, subjective astigmatism, videokeratoscopic measurements (that is, astigmatism, effective refractive power, corneal uniformity index, asphericity, predicted corneal acuity, and refractive power symmetry index), ${ }^{78}$ intraocular pressure, graft central thickness, postoperative endothelial cell density, and postoperative percentage endothelial cell loss were analysed using the KruskalWallis one way ANOVA and Wilcoxon rank sum test. As multiple comparisons were made, we also used the method reported by Edwards to correct their significance levels for the total number of comparisons. ${ }^{9}$ The likelihood for posterior capsule opacification in patients with clear transplant was analysed with the KaplanMeier method and log rank test.

\section{Results}

An intact continuous tear capsulotomy was performed in $62.5 \%$ of cases (25/40). Out of 25 patients with an intact capsulorhexis, phacoemulsification was used in 13 patients $(52 \%)$ whereas the entire nucleus passed through the capsulorhexis in the remaining 12 patients $(48 \%) ; 23$ posterior chamber IOLs were placed in the capsular bag and 13 were placed in the ciliary sulcus. A tear in the posterior capsule occurred in four patients $(10.0 \%)$. An anterior chamber IOL was placed in three patients and no lens was placed in a patient with pre-existing severe iris damage.

A capsulorhexis was performed in 57\% (12/ 21 ) of the patients operated on under peribulbar anaesthesia and $68 \%(13 / 19)$ of those operated on under general anaesthesia $(\mathrm{p}=$ $0.68)$. A tear in the posterior capsule occurred in $14 \%(3 / 21)$ of the patients operated on under peribulbar anaesthesia and 5\% (1/19) of those operated on under general anaesthesia ( $\mathrm{p}$ $=0.67)$. The IOL was placed within the bag in $52 \%(11 / 21)$ of the patients operated on under peribulbar anaesthesia and $63 \%(12 / 19)$ of those operated on under general anaesthesia $(\mathrm{p}$ $=0.60)$.

Table 2 shows the overall results. Graft failure occurred in 13 out of the 40 eyes. Allograft rejection was the leading cause of failure

Table 3 Comparison of the group of patients with in the bag IOL with the group of patients with ciliary sulcus IOL. Visual acuity, astigmatism, videokeratoscopic measurements, intraocular pressure, graft thickness, and endothelial cell density were measured excluding patients with graft failure

\begin{tabular}{llll}
\hline & $\begin{array}{l}\text { Bag group } \\
(n=23)\end{array}$ & $\begin{array}{l}\text { Sulcus group } \\
(n=13)\end{array}$ & $p$ Value \\
\hline Two year graft survival estimate (\%) & 82.6 & 64.5 & 0.17 (NS) \\
Spectacle corrected visual acuity at 12 months (mean (SD)) & $\mathbf{0 . 4 6 ( 0 . 2 1 )}$ & $\mathbf{0 . 2 9}(\mathbf{0 . 0 8})$ & $\mathbf{0 . 0 4}$ \\
Spherical equivalent (D) at 12 months (mean (SD)) & $-2.7(3.5)$ & $-4.1(3.0)$ & 0.31 (NS) \\
Spectacle corrected visual acuity at 18 months (mean (SD)) & $0.50(0.28)$ & $0.26(0.11)$ & 0.15 (NS) \\
Subjective astigmatism (D) at 12 months (mean (SD)) & $3.83(1.77)$ & $4.21(2.36)$ & 0.64 (NS) \\
Subjective astigmatism (D) at 18 months (mean (SD)) & $3.50(1.73)$ & $3.60(0.55)$ & 0.77 (NS) \\
Videokeratoscopic astigmatism (D) (mean (SD)) & $4.47(3.07)$ & $6.06(4.35)$ & 0.73 (NS) \\
Predicted corneal acuity (mean (SD)) & $0.98(0.63)$ & $0.98(0.90)$ & 0.83 (NS) \\
Refractive power symmetry index (D) (mean (SD)) & $1.91(1.08)$ & $3.57(0.45)$ & 0.23 (NS) \\
Effective refractive power (D) (mean (SD)) & $45.28(2.52)$ & $47.18(2.26)$ & 0.31 (NS) \\
Postoperative maximum IOP (mm Hg) (mean (SD)) & $18.1(5.9)$ & $25.6(13.3)$ & 0.06 (NS) \\
Postoperative graft thickness ( $\mu$ m) at 12 months (mean (SD)) & $548(44)$ & $560(60)$ & $0.58(\mathrm{NS})$ \\
Postoperative graft thickness ( $\mu$ m) at 18 months(mean (SD)) & $\mathbf{5 5 2 ( 2 7 )}$ & $\mathbf{6 5 0}(\mathbf{2 9})$ & $\mathbf{0 . 0 4}$ \\
Postoperative endothelial cell density (cells/mm (mean (SD)) & $1450(485)$ & $1460(359)$ & 0.75 (NS) \\
Postoperative percentage of endothelial cell loss (mean (SD)) & $35.3(23.2)$ & $29.2(22.4)$ & 0.35 (NS) \\
\hline
\end{tabular}

*Wilcoxon rank sum test. After correcting the significance levels for the total number of comparisons, all of the p values were above the 0.05 significance level. 


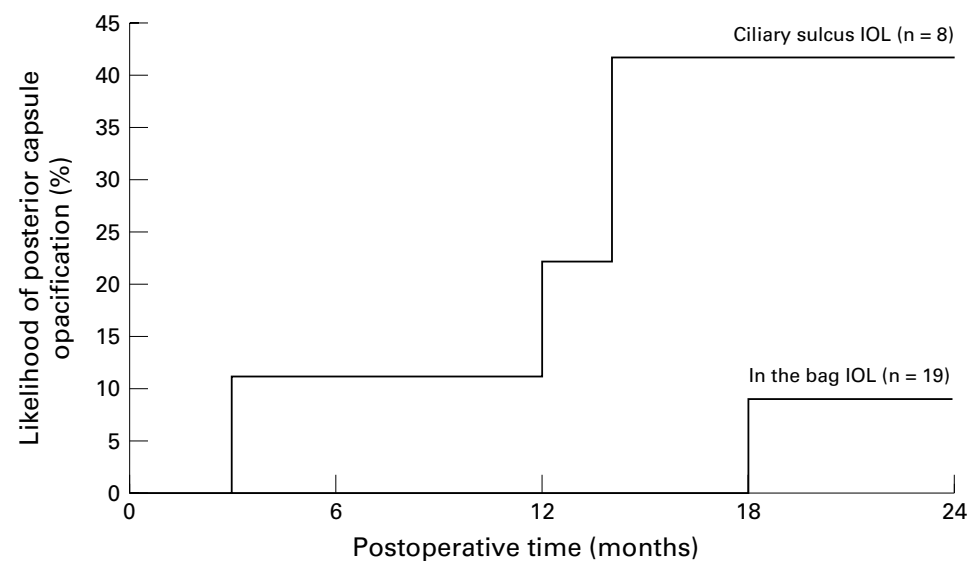

Figure 4 Influence of postoperative lens status on the likelihood for posterior capsule opacification in patients with clear transplant. Log rank: $p=0.07$. The numbers of patients at risk at the beginning of the curves are indicated at the end of the curves.

as it occurred in 11 eyes (84.6\%). Other causes of graft failure $(15.4 \%)$ included persistent epithelial defect (one eye) and glaucoma (one eye). At a univariate level, recipient rejection status ( $\mathrm{p}<0.0001$, Fig 1), postoperative lens status ( $\mathrm{p}=0.004$, Fig 2$)$, preoperative diagnosis ( $\mathrm{p}$ 0.0001, Fig 3), recipient age $(\mathrm{p}=$ $0.0001)$, and donor trephination size $(\mathrm{p}=$ 0.01 ) significantly influenced graft survival. In the multivariate Cox model, only the recipient rejection status ( $\mathrm{p}=0.04)$ significantly influenced graft survival. Donor age, endothelial density after preservation, preservation time, preoperative intraocular pressure, and suture method had no significant influence on graft survival.

Table 3 shows comparison of the group of patients with in the bag IOL (bag group, $\mathrm{n}=$ 23 ) with the group of patients with ciliary sulcus IOL (sulcus group, $n=13$ ). No significant difference between both groups was found considering the preoperative variables (Table 1). In patients with clear transplants, postoperative spectacle corrected visual acuity at 12 months was higher and postoperative graft thickness at 12 months was lower in the bag group than in the sulcus group. Elevated intraocular pressure (IOP) occurred in $26.1 \%$ $(6 / 23)$ of patients with in the bag IOL and $61.5 \%(8 / 13)$ of patients with ciliary sulcus IOL $(p=0.08)$. Figure 4 shows the likelihood for posterior capsule opacification in patients with clear transplant and posterior chamber IOL. Whereas posterior capsule opacification occurred later in the bag group than in the sulcus group, the difference between both groups did not reach the statistically significant threshold ( $p=0.07)$. Out of 27 patients with clear transplant and posterior chamber IOL, YAG laser capsulotomy was performed in five patients $(18.5 \%)$ an average of 15 (7) months after transplantation.

No expulsive haemorrhage, no endophthalmitis, and no retinal detachment occurred after these 40 triple procedures.

\section{Discussion}

In a single centre prospective study of 40 consecutive triple procedures, we found the 2 year graft survival estimate to be $66 \%$. Graft survival after triple procedure is reported to range from $60 \%$ to $100 \%$ in the literature. ${ }^{10-15}$ These studies generally include a high percentage of patients with Fuchs' dystrophy, ranging from $60 \%$ to $100 \%$. This results in high graft survival estimate. Two recent studies including only patients with Fuchs' dystrophy reported a $96 \%$ and $100 \%$ graft survival estimate. ${ }^{12} 15$ The present series includes 11 patients $(27 \%)$ with Fuchs' dystrophy. It is notable that no graft failure occurred in these patients. On the other hand, the present series includes a large number of fair prognosis transplants (recipients with a vascularised cornea or a history of allograft rejection). In fact, allograft rejection was the leading cause of graft failure accounting for $85 \%$ of failures. This high rate of immunological failure is responsible for the relatively low 2 year graft survival estimate (66\%) compared with recent studies. ${ }^{12}{ }^{15}$ It is noteworthy that $40 \%$ of our recipients were high risk recipients, and that the recipient rejection status strongly influenced graft survival. This influence of corneal vascularisation and history of allograft rejection on allograft rejection is in accordance with other studies. ${ }^{16-18}$ Considering the conclusions of two collaborative studies, HLA matching was not performed even for high risk recipients. ${ }^{19} 20$

Our goal was to perform a continuous tear capsulotomy open sky. This could be done in $63 \%$ of patients. No statistical difference was found in the percentage of cases with achieved capsulorhexis between patients operated on under peribulbar anaesthesia and patients operated on under general anaesthesia. When posterior vitreous pressure was high and the capsule torn towards the periphery, the capsulotomy was done in a can opener fashion. Another solution would have been to complete the capsulorhexis using scissors. However, we preferred placing a well centred large diameter IOL in the ciliary sulcus to placing a small diameter IOL in a capsular bag with sharp junctions which could result in later decentration of the IOL. Since a relatively small size capsulotomy was performed, large nuclei could not pass through the capsulorhexis in 13 out of 25 patients $(52 \%)$ with an intact capsulorhexis. In these patients phacoemulsification was useful to fragment and remove the nucleus. Finally, a well centred IOL could be placed within the capsular bag of 23 out of 25 patients with an intact capsulorhexis. For the remaining two patients, posterior vitreous pressure was too high and the capsular bag could not be filled with the viscoelastic substance. A large diameter IOL was then placed in the ciliary sulcus.

In the bag placement of the IOL gave better results than ciliary sulcus placement of the IOL. In fact, visual acuity at 12 months and graft thickness at 18 months were significantly better in the group of patients with in the bag IOL than in the group of patients with ciliary sulcus IOL. After correcting for the total number of comparisons, none of the observed differences between both groups was significant. However, correcting for the total number of comparisons implies that tests are independ- 
ent. The tests in the present analysis are most often dependent (that is, visual acuity at 12 months and visual acuity at 18 months are dependent variables) and the correction described by Edwards ${ }^{9}$ may be inappropriate. Anyway our results must be considered as a trend towards better visual acuity and better graft thickness with in the bag IOL. This may be considered as clinically relevant based on the results of cataract surgery. However, no randomisation was made between both groups. Only a further prospective and randomised study comparing triple procedures with in the bag placement of the IOL with triple procedures with ciliary sulcus placement of the IOL will be able to determine whether both procedures give significantly different results.

Many factors can influence visual acuity in pseudophakic patients after corneal transplantation: graft transparency, corneal anterior surface astigmatism and regularity, corneal posterior surface astigmatism and regularity, IOL position, posterior capsule opacification, vitreous transparency, macular condition. From our data, it is difficult to explain why visual acuity was better in the bag group. As we used $7 \mathrm{~mm}$ PMMA posterior chamber IOL in the sulcus group, postoperative IOL decentration was unlikely to occur. Currently, the exact level of graft transparency is clinically impossible to assess. We only can classify grafts as clear or opaque. Ciliary sulcus placement of the IOL could slightly impair graft transparency, vitreous transparency, and macular condition by inducing chronic low blood-aqueous barrier damage. Lastly, our study included more corneal scars, corneal ulcers, and interstitial keratitis in the sulcus group than in the bag group. However, the difference between both groups was not significant.

The influence of IOL placement on graft survival was complex. Graft survival was higher in patients with in the bag IOL than in patients with ciliary sulcus IOL, and higher in patients with ciliary sulcus IOL than in patients with anterior chamber IOL. At a univariate level, the overall effect ( 2 degrees of freedom) of IOL placement on graft survival was statistically significant, whereas it was not significant in the multivariate model. Furthermore, at a univariate level, comparison of the bag group with the sulcus group (1 degree of freedom) showed no significant difference between both groups. Further studies, including a larger number of patients and reporting the precise IOL placement, are needed to determine the actual influence of IOL placement on graft survival. We could hypothesise that in the bag placement of the IOL would reduce blood-aqueous barrier damage which would lead to reduced postoperative immunological reactions and to higher graft survival. ${ }^{21}$ This advantage would have to be considered in high risk patients. On the other hand, among patients with clear transplant, no significant difference in postoperative endothelial cell density and percentage of endothelial cell loss was found between both groups. Postoperative maximum intraocular pressure was lower in the group of patients with in the bag IOL than in the group of patients with ciliary sulcus IOL, but the difference between both groups did not reach the statistically significant threshold. Though no quantitative analysis of anterior synechiae was made, we observed more formation of anterior synechiae in the sulcus group than in the bag group. We found no significant difference in postoperative refraction between both groups. Lastly, no significant difference in videokeratoscopic measurements was found between both groups, demonstrating the absence of influence of IOL placement on corneal topographic features.

In conclusion, in the bag placement of the IOL during triple procedure results in better outcome of transplantation than ciliary sulcus placement of the IOL. Phacoemulsification allows removal of large nuclei through a $5 \mathrm{~mm}$ capsulorhexis without performing relaxing incisions out towards the periphery of the capsule.

This work was supported by the Fondation Claude Bernard pour le développement des recherches biologiques et médicales dans les Hôpitaux de l'Assistance Publique à Paris.

1 Pamel GJ, Taylor DM. Combined procedures. Long-term observations. In: Brightbill FS, ed. Corneal surgery. Theory, technique and tissue. St Louis: Mosby, 1993:177-83.

2 Alldredge CD, Alldredge OD. Penetrating keratoplasty and cataract extraction. In: Krachmer JH, Mannis MJ, Holland EJ, eds. Cornea. Surgery of the cornea and conjunctiva. St Louis: Mosby-Year Book, 1997:1593-601.

3 Groden LR. Continuous tear capsulotomy and phacoemulsification cataract extraction comined with penetrating keratoplasty. Refract Corneal Surg 1990;6:458-9.

4 Malbran ES, Malbran E, Buonsanti J, et al. Closed-system phacoemulsification and posterior chamber implant combined with penetrating keratoplasty. Ophthalmic Surg 1993; 24:403-6.

5 Borderie VM, Kantelip BM, Delbosc BY, et al. Morphology, histology and ultrastructure of human C31 organ-cultured corneas. Cornea 1995;14:300-10.

6 Borderie VM, Scheer S, Touzeau O, et al. Donor corneal tissue selection before penetrating keratoplasty. Br f Ophthalmol 1998;82:382-8.

7 Borderie VM, Laroche L. Measurement of irregular astigmatism using semimeridian data from videokeratographs. F Refract Surg 1996;12:595-600.

8 Holladay JT. Corneal topography using the Holladay diagnostic summary. 7 Cataract Refract Surg 1997;13:20921 .

9 Edwards JH. HL-A and disease. The detection of associations. F Immunogenet 1974;1:249-57.

10 Crawford GJ, Stulting RD, Waring GO, et al. The triple procedure. Analysis of outcome, refraction, and intraocular lens power calculation. Ophthalmology 1986;93:817-24.

11 Meyer RF, Musch DC. Assessment of success and complications of triple procedure surgery. Am $\mathcal{f}$ Ophthalmol 1987;104:233-40.

12 Pineros OE, Cohen EJ, Rapuano CJ, et al. Triple vs nonsimultaneous procedures in Fuchs' dystrophy and cataract. Arch Ophthalmol 1996;114:525-8.

13 Taylor DM, Khalig R, Maxwell R. Keratoplasty and intraocular lenses: current status. Ophthalmology 1979;86: $242-54$

14 Lee JR, Dohlman CH. Intraocular lens implantation in combination with keratoplasty. Ann Ophthalmol 1977;9: 513-8.

15 Serdarevic ON, Renard GJ, Pouliquen YP. Videokeratoscopy of recipient peripheral corneas in combined penetrating keratoplasty, cataract extraction, and lens implantation. Am 7 Ophthalmol 1996;122:29-37.

16 Boisjoly HM, Tourigny R, Bazin R, et al. Risk factors of corBoisjoly HM, Tourigny R, Bazin R, et al. Risk factors of
neal graft failure. Ophthalmology 1993;100:1728-35.

17 Boisjoly HM, Raynald R, Bernard PM, et al. Association between corneal allograft reactions and HLA compatibility. Ophthalmology 1990;97:1689-98.

18 Borderie VM, Lopez M, Vedie F, et al. ABO antigen blood group compatibility in corneal transplantation. Cornea 1997;16:1-6.

19 Collaborative Corneal Transplantation Studies Research Group. Effectiveness of histocompatibility matching in high-risk corneal transplantation. Arch Ophthalmol 1992; 110:1392-403.

20 Vail A, Gore SM, Bradley BA, et al. Influence of donor and histocompatibility factors on corneal graft outcome. Transplantation 1994;58:1210-7.

21 Keates RH, Rotchild EJ, Bloom R. Endocapsular triple procedure-a new triple procedure technique. $\mathcal{f}$ Cataract Refract Surg 1989;15:332-5. 